\title{
Characteristics that distinguish leiomyoma variants from the ordinary leiomyomas and recurrence risk
}

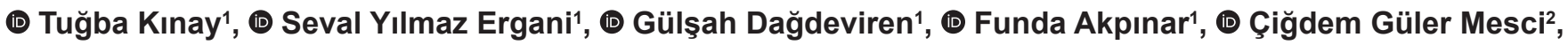

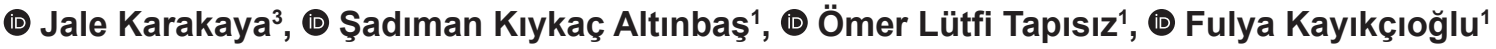

1 University of Health Sciences Turkey, Etlik Zubeyde Hanim Women's Health Training and Research Hospital, Clinic of Obstetrics and Gynecology, Ankara, Turkey

2University of Health Sciences Turkey, Etlik Zubeyde Hanim Women's Health Training and Research Hospital, Clinic of Pathology, Ankara, Turkey

3Hacettepe University Faculty of Medicine, Department of Biostatistics, Ankara, Turkey

Date submitted:

08.08.2020

Date accepted:

14.02.2021

Online publication date:

15.03.2022

\section{Corresponding Author:}

Tuğba Kınay, M.D., University of Health Sciences Turkey, Etlik Zubeyde Hanim Women's Health Training and Research Hospital, Clinic of Obstetrics and Gynecology, Ankara, Turkey tkinay@hotmail.com

ORCID:

orcid.org/0000-0001-5340-1025

Keywords: Cellular leiomyoma, leiomyoma variant, leiomyoma with bizarre nuclei, mitotically active leiomyoma, recurrence

\begin{abstract}
Aims: To evaluate the clinical characteristics that distinguish the leiomyoma variants from the ordinary ones, as well as the recurrence risk and patterns of these tumors.

Methods: This retrospective, case-control study included women diagnosed with uterine leiomyoma between 2009 and 2019 at a tertiary referral center in Turkey. The clinical characteristics of patients diagnosed with cellular leiomyoma $(\mathrm{CL})$, leiomyoma with bizarre nuclei (LBN), mitotically active leiomyoma (MAL), and ordinary leiomyoma (OL) were examined. Disease recurrence and patterns of variants were evaluated.
\end{abstract}

Results: Among the 1,581 women with uterine leiomyoma, the incidence of $C L, L B N$, and $M A L$ were $2.9 \%, 1.2 \%$, and $1.3 \%$, respectively. The occurrence for a single mass $(63 \%$ in $C L, 78.9 \%$ in LBN, $61.9 \%$ in $M A L, 38.8 \%$ in $\mathrm{OL} ; \mathrm{p}=0.001$ ) was more common, and the diagnosis of intramural leiomyoma (67.4\% in $\mathrm{CL}, 73.7 \%$ in $\mathrm{LBN}, 71.4 \%$ in $\mathrm{MAL}, 90.8 \%$ in $\mathrm{OL} ; \mathrm{p}=0.002$ ) was less frequent in the leiomyoma variant groups compared with the OL group. Recurrent leiomyosarcoma was observed in one woman with MAL.

Conclusions: This study showed that single mass rate was higher and intramural leiomyoma rate was lower in leiomyoma variants than in ordinary variants. Although rare, leiomyoma variants carry a risk of malignant recurrence after hysterectomy, suggesting the need for longterm follow-up.

\section{Introduction}

Leiomyoma variants are rare pelvic tumors. The variant rate is $1-4 \%(1,2)$. Cellular leiomyoma $(C L)$, leiomyoma with bizarre nuclei (LBN) (atypical leiomyoma, symplastic leiomyoma, bizarre leiomyoma), and mitotically active leiomyoma (MAL) are the three variants. These tumors have some leiomyosarcoma
(LMS) characteristics but do not have all the characteristics together. MAL has increased mitotic count of 5-20 mitotic figures per 10 high power fields but no nuclear atypia or tumor necrosis. LBN has moderate-to-severe nuclear atypia with low mitotic counts and no coagulative tumor cell necrosis (3). In the case of $\mathrm{CL}$, there is increased cellularity of the lesion compared to the surrounding myometrial tissue $(4,5)$. 
Leiomyoma variants have similar symptoms and findings in the pelvic examinations of patients with ordinary leiomyoma $(\mathrm{OL})$ and LMS. Immunohistochemistry, molecular-genetic analysis, and imaging techniques have a limited benefit for the differential diagnosis of these uterine mesenchymal tumors (6-9). It was reported that LMS and bizarre leiomyoma had significant overlapping staining patterns and immunoreactivity for p16, p53, and Ki-67 (7). Atypical leiomyoma and LMS share similar microRNA signatures (8). MED12 mutations were common in both $\mathrm{OL}$ and MAL (8). Magnetic resonance imaging (MRI), positron emission tomography and computed tomography have a limited utility to differentiate leiomyoma variants from $\mathrm{OL}$ and LMS (9). A definitive diagnosis is still currently carried out by a histopathological examination of myomectomy or hysterectomy specimens. Whether leiomyoma variants differ from one another in terms of clinical characteristics and behavior patterns is also unclear.

The management of variants after diagnosis is currently similar to the management of OL. However, the recurrence risk of these non-malignant variants has not been fully characterized yet, and rare malignant recurrent cases have been reported previously (10-12). Considering this knowledge, this study aims to evaluate the clinical characteristics that distinguish the leiomyoma variants from the ordinary ones, as well as the recurrence risk and patterns of these tumors.

\section{Methods}

In this retrospective study, medical records were reviewed for women who underwent hysterectomy or myomectomy due to a preoperative diagnosis of leiomyoma between January 2009 and August 2019 at a tertiary referral center. Written informed consent that allowed the use of their medical records in scientific research was obtained from all participants before the surgery. The study protocol was approved by the Institutional Review Board of University of Health Sciences Turkey, Etlik Zubeyde Hanim Women's Health Training and Research Hospital (protocol number: 2019/15, date: 10.10.2019) and complied with the Helsinki Declaration including current revisions.

The histopathological reports of the participants were reviewed. Women with $\mathrm{OL}$ and leiomyoma variants were included in the study. Women with adenomyosis, smooth muscle tumors of uncertain malignant potential (STUMP), uterine sarcomas, and concomitant endometrial, ovarian, and cervical malignancies were excluded from the study. According to the histopathological diagnosis, the study population was divided into four groups: the CL group, the LBN group, the MAL group, and the OL group. The control group consisted of OL cases. The sample size of the control group was determined to be approximately twice that of the largest variant group. The demographic, clinical, and histopathological characteristics of the four groups were compared.
Postmenopausal status was defined as no menstrual bleeding within (at least) one year before the operation in the absence of amenorrhea due to other reasons (such as intrauterine synechia, hyperprolactinemia, etc.). Regular or irregular bleeding, heavy and prolonged bleeding and intermenstrual bleeding were all identified as abnormal uterine bleeding. Surgery indications were described as previously reported $(4,13)$.

The number of leiomyomas, as well as their size and localization, were determined according to histopathological reports in women who underwent hysterectomy. These details were also determined on the basis of ultrasonography reports in women who underwent myomectomy or hysteroscopic resection. The presence of atypia, degeneration, and necrosis in the surgical specimen was noted on the basis of information from the histopathological reports. Histopathologically confirmed recurrence according to reoperation reports was accepted as recurrence.

\section{Statistical Analysis}

All statistical analyses were performed using Statistical Package for the Social Sciences (SPSS) Statistics for Windows (Version 23.0, Armonk, NY: IBM Corp., 2015). Normality for continuous variables was checked using the ShapiroWilk test. The descriptive statistics were expressed as a frequency and mean \pm standard deviation or median (minimummaximum). One-way ANOVA or the Kruskal-Wallis test was used to compare the differences among the groups where appropriate. Tukey test or the Dunn test was used for post-hoc analyses. The chi-square test or Fisher exact tests was used to compare the differences in categorical variables. A $p<0.05$ was accepted statistically significant.

\section{Results}

The medical records of 1,638 women with a preoperative diagnosis of uterine leiomyoma were evaluated. Nine women with LMS, 14 women with STUMP, two women with a mixed stromal tumor, four women with endometrial stromal sarcoma, 26 women with adenomyosis, and 2 women with concomitant ovarian carcinoma were excluded from the study. A total of 86 women diagnosed with leiomyoma variants (46 with $\mathrm{CL}, 19$ with LBN, and 21 with MAL), as well as 98 women with OL were included in the study. The participants' recruitment is as shown in Figure 1. Of the 1,581 women with uterine leiomyoma, the incidence of $\mathrm{CL}, \mathrm{LBN}$, and $\mathrm{MAL}$ were $2.9 \%, 1.2 \%$, and $1.3 \%$, respectively.

As shown in Table 1, the demographic and clinical characteristics of the four groups were similar except in age. The mean age of the OL group was higher than the LBN group $(46.5 \pm 5.8$ y vs. $42.3 \pm 6.7 \mathrm{y}, \mathrm{p}=0.037)$. Most women included in the study were in the premenopausal period. 
The postmenopausal rates of the groups were not significantly different. Ultrasonographic and histopathological findings of the women did not reveal any significant difference between the four groups in terms of tumor size and rates of degeneration and necrosis in leiomyoma specimens (Table 2). The median number of leiomyomas was higher in the OL group [3 (1-9)] than in the others [1 (1-7) in the CL group, $1(1-7)$ in the LBN group, and $1(1-6)$ in the MAL group] $(p<0.001)$. The single mass

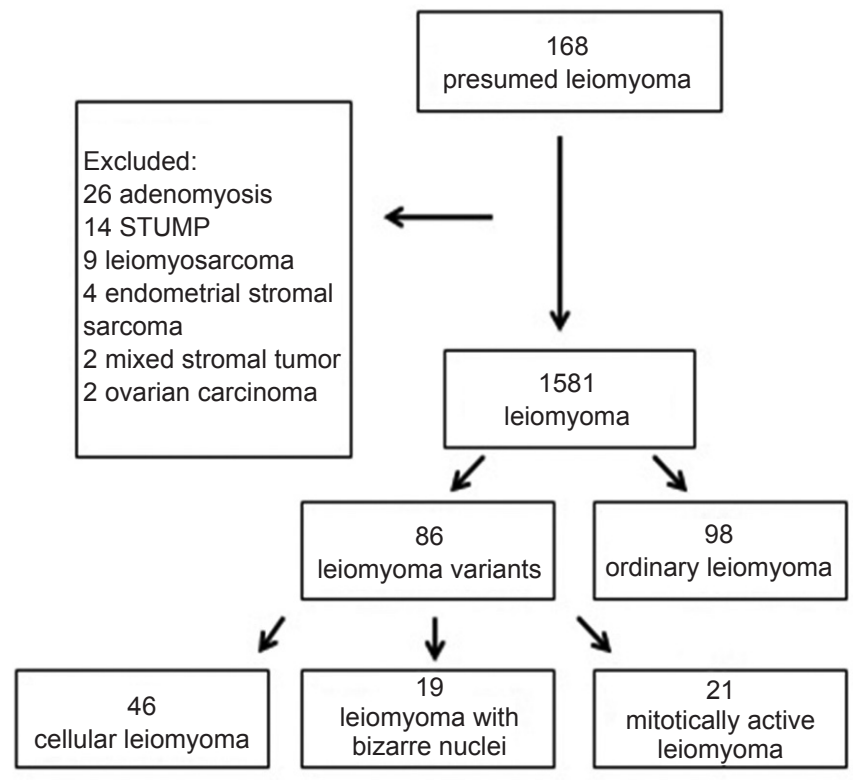

Figure 1. Flowchart of participants

STUMP: Smooth muscle tumors of uncertain malignant potential rate was higher in the leiomyoma variant groups than in the OL group (63\% in CL, $78.9 \%$ in LBN, $61.9 \%$ in MAL, $38.8 \%$ in $\mathrm{OL} ; \mathrm{p}=0.001)$. The rate of intramural leiomyoma was higher in the OL group than in the others $(90.8 \%$ in the OL group, $67.4 \%$ in the CL group, $73.7 \%$ in the LBN group, and $71.4 \%$ in the MAL group; $p=0.002$ ). The rates of submucosal and subserous leiomyoma of the four groups were similar.

Follow-up records of 73 women with leiomyoma variants were available. The mean follow-up period was $14.4 \pm 17.6$ months with a range of 1-72 months. During the follow-up period, recurrence was observed in one woman with the MAL. She was 46 years old. She underwent a total abdominal hysterectomy and bilateral salpingo-oophorectomy due to an $18 \mathrm{~cm}$ in diameter degenerated myoma. The histopathological diagnosis of the hysterectomy specimen was MAL. In the follow-up visit 15 months after surgery, multiple semisolid recurrent masses were observed in the abdominal MRI examination. Tumoral debulking was carried out in the second operation. LMS was detected in a histopathological diagnosis of the recurrent mass. The length of survival for the woman-administered chemotherapy after the second operation was 32 months.

\section{Discussion}

The study results show that no significant demographic and clinical characteristics differentiated the three leiomyoma variants from the other. However, the women with OL were significantly older than the women with LBN when they underwent surgery. Ultrasonographic and histopathological findings showed that the leiomyoma variants tended to be a single mass, whereas

\begin{tabular}{|c|c|c|c|c|c|}
\hline & $C L(n=46)$ & LBN $(n=19)$ & MAL $(n=21)$ & OL $(n=98)$ & $p$ value \\
\hline Age (years), mean $\pm S D$ & $44.7 \pm 7.2$ & $42.3 \pm 6.7$ & $43.5 \pm 5.1$ & $46.5 \pm 5.8$ & $0.017^{*}$ \\
\hline Gravidity, median (IQR) & $3(0-8)$ & $3(0-5)$ & $2(1-8)$ & $3(0-9)$ & 0.581 \\
\hline Parity, median (IQR) & $2(0-6)$ & $2(0-4)$ & $2(1-4)$ & $2(0-7)$ & 0.926 \\
\hline Postmenopausal, n (\%) & $4(8.7)$ & $1(5.3)$ & $0(0)$ & $7(7.1)$ & 0.689 \\
\hline Irregular menses, $\mathrm{n}(\%)$ & $20(43.5)$ & $11(57.9)$ & $14(66.7)$ & $50(53.2)$ & 0.330 \\
\hline Operation indication & & & & & 0.252 \\
\hline Symptomatic leiomyoma, n (\%) & $39(84.8)$ & $16(84.2)$ & $21(100)$ & $86(87.8)$ & \\
\hline Enlarging leiomyoma, $\mathrm{n}(\%)$ & $7(15.2)$ & $3(15.8)$ & $0(0)$ & $12(12.2)$ & \\
\hline \multicolumn{6}{|l|}{ Symptom } \\
\hline Abnormal uterine bleeding, $\mathrm{n}(\%)$ & $31(67.4)$ & $12(63.2)$ & $19(90.5)$ & $69(70.4)$ & 0.190 \\
\hline Postmenopausal bleeding, n (\%) & $1(2.2)$ & $0(0)$ & $0(0)$ & $0(0)$ & 0.467 \\
\hline Pelvic pain, $\mathrm{n}(\%)$ & $10(21.7)$ & $5(26.3)$ & $3(14.3)$ & $17(17.3)$ & 0.710 \\
\hline Urinary symptom, $\mathrm{n}(\%)$ & $0(0)$ & $1(5.3)$ & $0(0)$ & $1(1.0)$ & 0.328 \\
\hline $\begin{array}{l}\text { Preoperative hemoglobin (gr/dL), median } \\
\text { (IQR) }\end{array}$ & $12.2(5.8-15.6)$ & $11.8(9.6-14.9)$ & $11.6(7.7-14.1)$ & $12.2(5.8-15.2)$ & 0.500 \\
\hline \multicolumn{6}{|c|}{$\begin{array}{l}\text { *Comparison of } C L \text { and } L B N(p=0.497) \text {, comparison of } C L \text { and } M A L(p=0.878) \text {, comparison of } C L \text { and } O L(p=0.355) \text {, comparison of } L B N \text { and } M A L(p=0.935) \text {, } \\
\text { comparison of } \angle B N \text { and } O L(p=0.037) \text {, comparison of MAL and } O L(p=0.177) \text {. } \\
C L \text { : Cellular leiomyoma, } L B N \text { : Leiomyoma with bizarre nuclei, MAL: Mitotically active leiomyoma, OL: Ordinary leiomyoma, SD: Standard deviation, IQR: Interquartile } \\
\text { range }\end{array}$} \\
\hline
\end{tabular}




\begin{tabular}{|c|c|c|c|c|c|}
\hline & $C L(n=46)$ & LBN $(n=19)$ & MAL $(n=21)$ & OL $(n=98)$ & $p$ value \\
\hline Leiomyoma size, median (IQR) & $8(1.5-20)$ & $8(2.5-17)$ & $7.5(1-9)$ & $7.5(2.5-18.5)$ & 0.529 \\
\hline Single leiomyoma, n (\%) & $29(63.0)$ & $15(78.9)$ & $13(61.9)$ & $38(38.8)$ & 0.001 \\
\hline \multicolumn{6}{|l|}{ Leiomyoma localization, n (\%) } \\
\hline Intramural, n (\%) & $31(67.4)$ & $14(73.7)$ & $15(71.4)$ & $89(90.8)$ & $0.002^{*}$ \\
\hline Subserous, n (\%) & $8(17.4)$ & $4(21.0)$ & $1(4.8)$ & $29(29.6)$ & 0.065 \\
\hline Degeneration, n (\%) & $9(20.0)$ & $5(26.3)$ & $2(9.5)$ & $12(12.2)$ & 0.284 \\
\hline Atypia, n (\%) & $0(0)$ & $19(100)$ & $0(0)$ & $0(0)$ & $<0.001$ \\
\hline Necrosis, n (\%) & $1(2.2)$ & $0(0)$ & $0(0)$ & $0(0)$ & 0.467 \\
\hline
\end{tabular}

ordinary cases had multiple leiomyomas. The rate of intramural mass was also higher in ordinary cases than in the leiomyoma variants. In this series, one woman with MAL had a recurrence diagnosed as LMS.

Like previous reports, the incidence of leiomyoma variants was less than $5 \%$ in our series $(1,2,14)$. As previously reported, most women with leiomyoma variants were in the fifth decade of their life and in the premenopausal period when they underwent surgery. This was the same for the OL cases $(4,8,12,15)$. Symptoms of leiomyoma variants were also similar to OL cases in our series. Unlike our findings, Taran et al. (4) reported that menometrorrhagia and pelvic pressure symptoms were more likely in women with $\mathrm{CL}$ than OL.

Leiomyoma variants usually appear as a single mass $(4,8,13)$. In the Taran et al. (4) series, one leiomyoma was found amongst the CL cases and three were found amongst the women with OL, as was the case in our series. Rothmund et al. (13) reported the $51.3 \%$ rate of single uterine masses in women with $\mathrm{CL}$. We also found that the rate of the intramural tumor was lower in leiomyoma variants than in the OL cases. Prayson and Hart (16) reported that more than $60 \%$ of the MAL cases were submucosal. In our series, the rate of submucosal MAL was $23.8 \%$, and it was not significantly different from the other groups.

The evolution of minimally invasive and uterine-conserving therapies requires a better understanding of the clinical behavior of leiomyoma variants. Although leiomyoma variants are accepted as benign pelvic masses, previous reports suggest that these tumors have a malign behavior. Molecular genetic studies showed that transcriptional profiles of CL with a $1 p$ deletion were more likely to those of LMS than to profiles of OL on hierarchical cluster analysis (17). A metastasis of CL to the rib and vertebra three years after myomectomy (18), a pulmonary metastasis of CL 10 years after vaginal hysterectomy (4), and the recurrence of LBN (10) and MAL (11) as LMS were also reported previously. Cooney et al. (10) reported an atypical leiomyoma case recurring as LMS in the vagina 22 months after laparoscopic hysterectomy with bilateral salpingo-oophorectomy. Gregová et al. (12) reported two LMS cases eight months after hysterectomy and seven years after myomectomy due to LBN. Kim et al. (11) reported a malignant recurrence of MAL with hyaline degeneration after a total hysterectomy. LMS was detected seven months after the first operation in a 68-year-old postmenopausal woman. The recurrent variant cases reported in the literature are shown in Table 3 (4,10-13,15,18-22). In our series, the recurrent case was a premenopausal woman. She was 46 years old and underwent a total hysterectomy due to MAL with degenerative changes. LMS occurred 15 months after the surgery. Based on previous reports and current findings, close postoperative follow-up appointments are necessary for patients with leiomyoma variants, especially those with degenerative changes.

The retrospective design, incomplete data such as the amount of decrease in hemoglobin levels that lead to operation decision and histopathological evaluation of surgical specimens by different pathologists are the limitations of this study. Nevertheless, the examination of all surgical specimens by experienced gynecopathologists at a single tertiary care center compensated for these limitations. The relatively small sample size of the variant groups and short follow-up periods were the other limitations of the study. Prospective studies with a larger series and longer follow-up periods are needed to confirm the presented results. A better understanding of the clinical characteristics and behavior of these tumors will lead to choosing an adequate surgical treatment modality and follow-up period. 


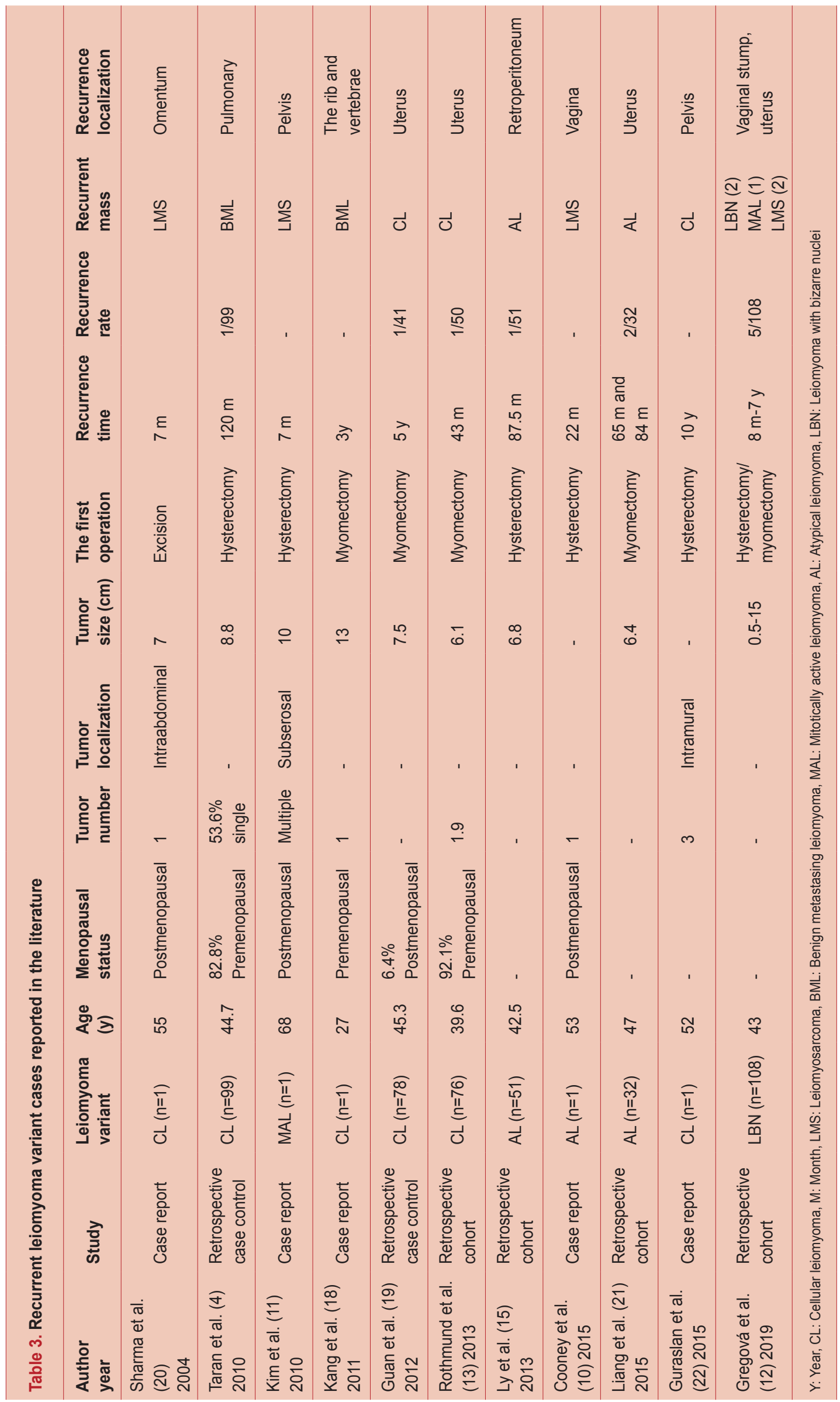




\section{Conclusion}

In conclusion, the leiomyoma variants had a higher rate of single masses and a lower rate of intramural localization than the ordinary ones. It should be kept in mind that there may be a leiomyoma variant in women with a single leiomyoma other than intramural localization. Additionally, this study showed that the leiomyoma variants have a risk of malign recurrence after hysterectomy, even if this is rare. Long-term follow-up will be needed after hysterectomies or myomectomies are carried out due to these rare tumors.

\section{Ethics}

Ethics Committee Approval: The study were approved by the Institutional Review Board of University of Health Sciences Turkey, Etlik Zubeyde Hanim Women's Health Training and Research Hospital, of Local Ethics Committee (protocol number: 2019/15, date: 10.10.2019).

Informed Consent: Retrospective study.

Peer-review: Externally and internally peer-reviewed.

\section{Authorship Contributions}

Concept: T.K., F.K., Design: T.K., S.Y.E., F.K., Ç.G.M., Ş.K.A., Ö.L.T., Data Collection or Processing: T.K., S.Y.E., G.D., F.A., Analysis or Interpretation: Ç.G.M., J.K., Ş.K.A., Ö.L.T., F.KÜ., Literature Search: T.K., S.Y.E., G.D., F.A., Writing: T.K., J.K.

Conflict of Interest: No conflict of interest was declared by the authors.

Financial Disclosure: The authors declared that this study received no financial support.

\section{References}

1. Damasco MR, Chan PK, Slonim M, Ang WC, Healey MG. Incidence of Malignancy and Myoma Variants at Surgery for Presumed Benign Symptomatic Myomas. J Minim Invasive Gynecol. 2017;24:659-664.

2. Seidman MA, Oduyebo $T$, Muto MG, Crum $C P$, Nucci MR, Quade BJ. Peritoneal dissemination complicating morcellation of uterine mesenchymal neoplasms. PLoS One. 2012; 7:e50058.

3. Bell SW, Kempson RL, Hendrickson MR. Problematic uterine smooth muscle neoplasms. A clinicopathologic study of 213 cases. Am J Surg Pathol. 1994;18:535-558.

4. Taran FA, Weaver AL, Gostout BS, Stewart EA. Understanding cellular leiomyomas: a case-control study. Am J Obstet Gynecol. 2010;203:109.

5. Ip PP, Tse KY, Tam KF. Uterine smooth muscle tumors other than the ordinary leiomyomas and leiomyosarcomas: a review of selected variants with emphasis on recent advances and unusual morphology that may cause concern for malignancy. Adv Anat Pathol. 2010;17:91-112.
6. D'Angelo E, Prat J. Diagnostic use of immunohistochemistry in uterine mesenchymal tumors. Semin Diagn Pathol. 2014;31:216-222.

7. Chen L, Yang B. Immunohistochemical analysis of p16, p53, and $\mathrm{Ki}-67$ expression in uterine smooth muscle tumors. Int $\mathrm{J}$ Gynecol Pathol. 2008;27:326-332.

8. Zhang Q, Ubago J, Li L, et al. Molecular analyses of 6 different types of uterine smooth muscle tumors: Emphasis in atypical leiomyoma. Cancer. 2014;120:3165-3177.

9. Arleo EK, Schwartz PE, Hui P, McCarthy S. Review of Leiomyoma Variants. AJR Am J Roentgenol. 2015;205:912-921.

10. Cooney EJ, Borowsky M, Flynn C. Case report: Atypical, 'symplastic' leiomyoma recurring as leiomyosarcoma in the vagina. Gynecol Oncol Rep. 2015;14:4-5.

11. Kim JH, Choi YJ, Kim DC, Lee SJ. Leiomyosarcoma arising in a patient with prior mitotically active leiomyoma. J Obstet Gynaecol Res. 2010;36:187-190.

12. Gregová $M$, Hojný J, Němejcová $K$, et al. Leiomyoma with Bizarre Nuclei: a Study of 108 Cases Focusing on Clinicopathological Features, Morphology, and Fumarate Hydratase Alterations. Pathol Oncol Res. 2020;26:1527-1537.

13. Rothmund R, Kurth RR, Lukasinski NM, et al. Clinical and pathological characteristics, pathological reevaluation and recurrence patterns of cellular leiomyomas: a retrospective study in 76 patients. Eur J Obstet Gynecol Reprod Biol. 2013;171:358-361.

14. Wilkinson N, Rollason TP. Recent advances in the pathology of smooth muscle tumours of the uterus. Histopathology. 2001;39:331-341.

15. Ly A, Mills AM, McKenney JK, et al. Atypical leiomyomas of the uterus: a clinicopathologic study of 51 cases. Am J Surg Pathol. 2013;37:643-649.

16. Prayson RA, Hart WR. Mitotically active leiomyomas of the uterus. Am J Clin Pathol. 1992;97:14-20.

17. Christacos NC, Quade BJ, Dal Cin P, Morton CC. Uterine leiomyomata with deletions of Ip represent a distinct cytogenetic subgroup associated with unusual histologic features. Genes Chromosomes Cancer. 2006;45:304-312.

18. Kang MW, Kang SK, Yu JH, et al. Benign metastasizing leiomyoma: metastasis to rib and vertebra. Ann Thorac Surg. 2011;91:924-926.

19. Guan R, Zheng W, Xu M. A retrospective analysis of the clinicopathologic characteristics of uterine cellular leiomyomas in China. Int J Gynaecol Obstet. 2012;118:52-55.

20. Sharma P, Chaturvedi KU, Gupta R, Nigam S. Leiomyomatosis peritonealis disseminata with malignant change in a postmenopausal woman. Gynecol Oncol. 2004;95:742-745.

21. Liang $Y$, Zhang $X$, Chen $X$, Lü $W$. Diagnostic value of progesterone receptor, p16, p53 and $\mathrm{pHH} 3$ expression in uterine atypical leiomyoma. Int J Clin Exp Pathol. 2015;8:71967202.

22. Guraslan H, Senturk MB, Helvacioglu C, Aktas AG, Yasar L. Recurrent cellular leiomyoma 10 years after total abdominal hysterectomy. J Obstet Gynaecol. 2015;35:854-855. 